\title{
Search for Close-in Planets around Evolved Stars with Phase-curve variations and Radial Velocity Measurements
}

\author{
Teruyuki Hirano ${ }^{1}$, Bun'ei Sato ${ }^{1}$, Kento Masuda ${ }^{2}$, Othman Michel \\ Benomar $^{2}$, Yoichi Takeda ${ }^{3}$, Masashi Omiya ${ }^{3}$ and Hiroki Harakawa ${ }^{3}$ \\ ${ }^{1}$ Tokyo Institute of Technology, \\ 2-12-1 Ookayama, Meguro-ku, Tokyo 152-8551, Japan \\ email: hirano@geo.titech.ac.jp \\ ${ }^{2}$ The University of Tokyo \\ ${ }^{3}$ National Astronomical Observatory
}

\begin{abstract}
Tidal interactions are a key process to understand the evolution history of close-in exoplanets. But tidals still have a large uncertainty in their prediction for the damping timescales of stellar obliquity and semi-major axis. We have worked on a search for transiting giant planets around evolved stars, for which few close-in planets were discovered. It has been reported that evolved stars lack close-in planets, which is often attributed to the tidal evolution and/or engulfment of close-in planets by the hosts. Meanwhile, Kepler has detected a certain fraction of transiting planet candidates around evolved stars. Confirming the planetary nature for these candidates is especially important since the comparison between the occurrence rates of close-in planets around main sequence stars and evolved stars provides a unique opportunity to discuss the final stage of close-in planets. With the aim of confirming KOI planet candidates around evolved stars, we measured precision radial velocities (RVs) for evolved stars with transiting planet candidates using Subaru/HDS. We also developed a new code which simultaneously models and fits the observed RVs and phase-curve variations in the Kepler data (e.g., transits, stellar ellipsoidal variations, and planet emission/reflected light). As a result of applying the global fit to KOI giants/subgiants, we confirmed two giant planets around evolved stars (Kepler-91 and KOI-1894), as well as revealed that KOI-977 is more likely a false positive.
\end{abstract}

Keywords. planets and satellites: individual (KOI-977, KOI-1894, KOI-2133)

\section{Introduction}

It has been reported that evolved stars lack close-in planets, which is often attributed to the tidal evolution and/or engulfment of close-in planets by the hosts. In the past year, we have worked on a search for giant planets around evolved stars, for which few close-in planets were discovered. We resort to both radial velocity (RV) measurements and analysis of "phase-curve variations" for planetary candidates detected by Kepler. Phase-curve variations are comprised of Doppler boosting, ellipsoidal variations, and reflected/emission light from the companion. From a precise phase-folded light-curve, we can extract the system parameters such as companion's mass and scaled semi-major axis, etc. Among the three effects above, ellipsoidal variation is particularly important for close-in planets around evolved stars, since its amplitude is approximately proportional to the cube of stellar radius. Combining the analysis of phase-curves (including transits) with an RV measurement would enable a more precise determination of system parameters. We apply this technique to three KOI (Kepler Object of Interest) systems below. 


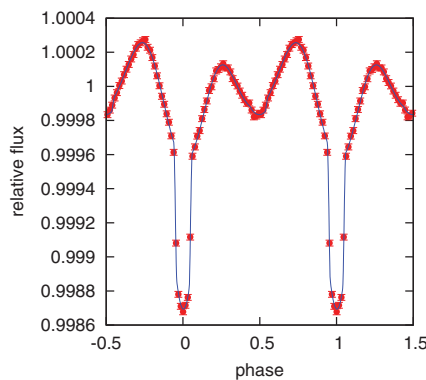

Figure 1. Phase-curve for KOI-977

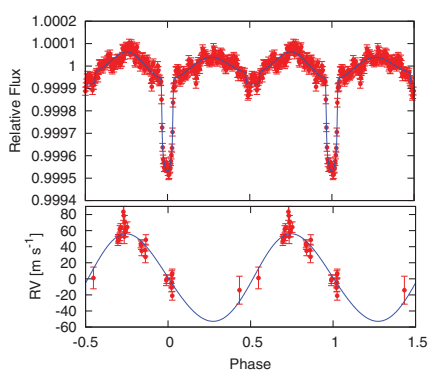

Figure 2. Phase-curve for Kepler-91

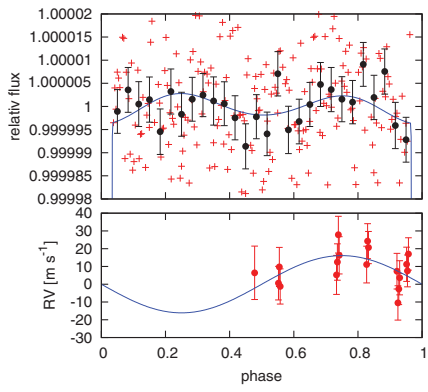

Figure 3. Phase-curve for KOI-1894

\section{Analyses and Results}

KOI-97\%: False Positive. We first focused on KOI-977, which is reported to be a red giant in the Kepler Input Catalog (KIC), with a close-in $(P=1.35$ days $)$ giant planet candidate. In order to confirm the planetary nature of KOI-977.01, we performed 1) RV measurements, 3) asteroseismic analysis, and 4) phase-curve analysis of Kepler data. Consequently, though the folded light-curve exhibits a clear pattern of phase-curve variations, our stellar analyses by spectroscopy and asteroseismology suggest that the estimated radius is too large for a star with a close-in planet; the estimated semi-major axis of the planet candidate KOI-977.01 is $\sim 0.027 \mathrm{AU}$, which is smaller than the stellar radius $\left(R_{\star} \sim 0.093 \mathrm{AU}\right)$. Combining this fact with the small RV variation revealed by the Subaru/HDS observation, KOI-977.01 is very likely a false positive, meaning that the transit-like signal is caused by an eclipsing binary, which is not identical to the red giant for which we measured stellar radius and RVs. On the assumption that KOI-977 is comprised of a red giant and an eclipsing binary, we searched for a solution to the observed phase-curve. Figure 1 shows the observed phase-curve (red) and its best-fit model (blue) by our MCMC fit. The resulting mass and radius ratios of the eclipsing binary range between $0.11<M_{1} / M_{2}<0.28$ and $0.13<R_{1} / R_{2}<0.17$, respectively. These values correspond to those of an F star and an early M star (Hirano et al. 2015).

Kepler-91 and KOI-1894: Real Planets. Two other systems were targeted by our search for giant planets around evolved stars: Kepler-91 (KOI-2133) and KOI-1894 (Kepler-91b was reported to be a real planet by Lillo-box et al. 2013 before our publication). These two stars have similar properties (e.g., $R_{\star}=6.3 R_{\odot}$ for Kepler-91 and $R_{\star}=8.6 R_{\odot}$ for KOI-1894) and similar planetary candidates (jovian planets with $P=6.2$ days and $P=5.3$ days, respectively). In order to gain the system parameters as accurately as possible, we combined the RV data by Subaru/HDS and Kepler public light-curve and performed a global analysis. The results of the fits confirmed the planetary nature for both candidates; The best-fit models as plotted in Figures 2 and 3 suggest that Kepler$91 \mathrm{~b}$ is a sub-Jupiter mass planet $\left(M_{p}=0.64 \pm 0.05 M_{J}\right)$ while KOI-1894b is more likely a super-Neptune planet $\left(M_{p}=0.14 \pm 0.08 M_{J}\right)$ (Sato et al. 2015).

\section{References}

Hirano, T., Masuda, K., Sato, B., Benomar, O., Takeda, Y., Omiya, M., Harakawa, H., \& Kobayashi, A. 2015, ApJ, 799, 9

Lillo-Box, J., Barrado, D., Moya, A., Montesinos, B., Montalban, J., Bayo, A., Barbieri, M., Regulo, C., Mancini, L., Bouy, H., \& Henning, T. 2014, A\&A, 562, A109

Sato, B., Hirano, T., Omiya, M., Harakawa, H., Kobayashi, A., Hasegawa, R., Takarada, T., Kawauchi, K., \& Masuda, K. 2015, ApJ, 802, 57 